\title{
Application of Fuzzy PID in Coordinated Control Object of Supercritical Unit
}

\author{
Lincang $\mathrm{Ju}^{1, \mathrm{a}}$,Guanggang Shang ${ }^{2, \mathrm{~b}}$,Yanggen Shi ${ }^{1, \mathrm{c}^{*}}$, Lei Wan ${ }^{1, \mathrm{~d}}$ \\ ${ }^{1}$ Xi'an Jiaotong University,28th Xianning West Rd, Xi'an 710049, China \\ ${ }^{2}$ Shandong Electric Power Engineering Consulting Institute Corp., 106th minziqian Rd,Jinan, China \\ ${ }^{1}$ lincang@mail.xjtu.edu.cn, ${ }^{2}$ Shangguanggang@sdepci.com, ${ }^{3,{ }^{*}}$ seek.season@stu.xjtu.edu.cn, \\ ${ }^{4}$ wanlei.2012@stu.xjtu.edu.cn
}

\begin{abstract}
Keywords: Coordinated control object; Decouple; Fuzzy; Adaptive; Controller
Abstract. Based on the model of coordinated control object, this paper firstly eliminates the strong coupling between the input and output variables using feedforward compensation decoupling method. The fuzzy adaptive PID controller, using the decoupled model, is designed and optimized for the main steam pressure channel and the intermediate point temperature channel of the coordinated control object. The simulation results show that, using the fuzzy adaptive PID controller optimized by quantum-behaved particle swarm optimization algorithm, the overshoot of controlled variable decreases and the adjustment time reduces in response to a step change. Furthermore, the control performance of PID controller is greatly improved.
\end{abstract}

\section{Introduction}

PID controller is the most widely used controller for supercritical unit at present. Meanwhile, to ensure the timeliness and effectiveness of PID controller, feedforward control technology is generally used in power plants ${ }^{[1]}$. PID controller is especially known for simple structure, ease of implementation and good robustness. Nevertheless, traditional PID controller cannot achieve the satisfactory control effect $^{[2]}$ when the controlled object is of strong nonlinear or has a long delay, because the traditional PID controller is linear. To adapt to the nonlinearity and time lag of controlled objects, we are suggested to take advantage of intelligent control methods like fuzzy control. These intelligent control methods can even be used without the awareness of precise mathematical models. However, pure intelligent control methods are usually weak on eliminating the steady-state error, which means high control precision could not be achieved. Thus, to promote the control performance of supercritical unit, as well as to achieve the security and reliability demand of power plants, we can carry out the strategy of combining fuzzy control and traditional PID control together, mixing the advantages of both. In this way, a significant difference can be made to improve the control performance of the units $^{[3]}$.

\section{Introduction of decoupled model of coordinated control object}

As there is no steam drum within once-through boiler used in supercritical unit, strong couplings exist between different control loops. When any of the three input parameters changes (the steam turbine control valve opening, the coal feeding quantity and the feedwater flow), all of the three output parameters would change accordingly (the unit power, the main steam pressure and the intermediate point temperature). From this perspective, the coordinated control object of supercritical unit could be treated as a coupled system with three inputs and three outputs. However, the control of the object becomes more difficult because of the coupled relationships between the inputs and the outputs.

To improve the control performance, we could decouple the coordinated control object with the help of decoupling theory. The feed-forward compensation decoupling method is famous for its simple principles as well as its good decoupling effect ${ }^{[4]}$. Thus, this method is adopted in this thesis. For coordinated control object of supercritical unit, the design of the feed-forward decoupling structure is shown in Figure 1. 


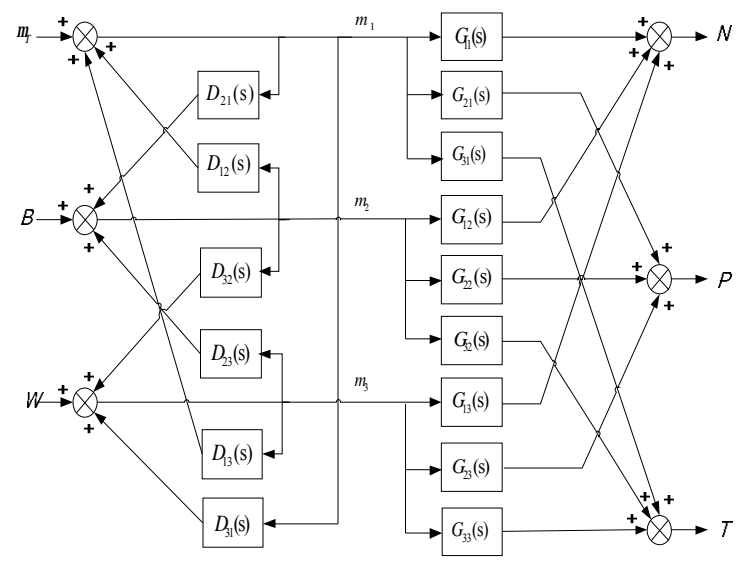

Figure.1 The feed-forward decoupling structure of coordinated control object of supercritical unit

If we assume these: the input vector is $U$, the input vector after decoupling compensation is $M$, the output vector is $Y$, the transfer function matrix is $G$, the decoupling matrix is $D$, we could get the follows:

$$
Y=G M=\left\{\begin{array}{l}
y_{1}=m_{1} G_{11}(s)+m_{2} G_{12}(s)+m_{3} G_{13}(s) \\
y_{2}=m_{1} G_{21}(s)+m_{2} G_{22}(s)+m_{3} G_{23}(s) \\
y_{3}=m_{1} G_{31}(s)+m_{2} G_{32}(s)+m_{3} G_{33}(s)
\end{array}\right.
$$

After the transformation:

$$
\left\{\begin{array}{l}
m_{1}=u_{1}+D_{12}(s) m_{2}+D_{13}(s) m_{3} \\
m_{2}=u_{2}+D_{21}(s) m_{1}+D_{23}(s) m_{3} \\
m_{3}=u_{3}+D_{31}(s) m_{1}+D_{32}(s) m_{2}
\end{array}\right.
$$

Namely:

$$
U=D M=\left\{\begin{array}{l}
u_{1}=m_{1}-D_{12}(s) m_{2}-D_{13}(s) m_{3} \\
u_{2}=m_{2}-D_{21}(s) m_{1}-D_{23}(s) m_{3} \\
u_{3}=m_{3}-D_{31}(s) m_{1}-D_{32}(s) m_{2}
\end{array}\right.
$$

The decoupling matrix is:

$$
D=\left[\begin{array}{ccc}
1 & G_{12}(s) / G_{11}(s) & G_{13}(s) / G_{11}(s) \\
G_{21}(s) / G_{22}(s) & 1 & G_{23}(s) / G_{22}(s) \\
G_{31}(s) / G_{33}(s) & G_{32}(s) / G_{33}(s) & 1
\end{array}\right]
$$

Thus:

$$
\left[\begin{array}{l}
y_{1} \\
y_{2} \\
y_{3}
\end{array}\right]=G D^{-1} U=\left[\begin{array}{ccc}
G_{11}(s) & 0 & 0 \\
0 & G_{22}(s) & 0 \\
0 & 0 & G_{33}(s)
\end{array}\right]\left[\begin{array}{l}
u_{1} \\
u_{2} \\
u_{3}
\end{array}\right]
$$

As we can see from the above equation, the decoupling relationships between the inputs and the outputs are eliminated through the decoupling network. That is, each output parameter is only affected by one corresponding input parameter.

\section{The fuzzy PID control system}

Combining conventional PID controller with fuzzy adaptive PID controller is the control strategy in this thesis. For the unit power channel, conventional PID control is adopted. And for main steam pressure channel and the intermediate point temperature whose response is slower, adaptive fuzzy PID control is adopted to improve the response rate of the system. The structure of the control system is shown in figure 2. 


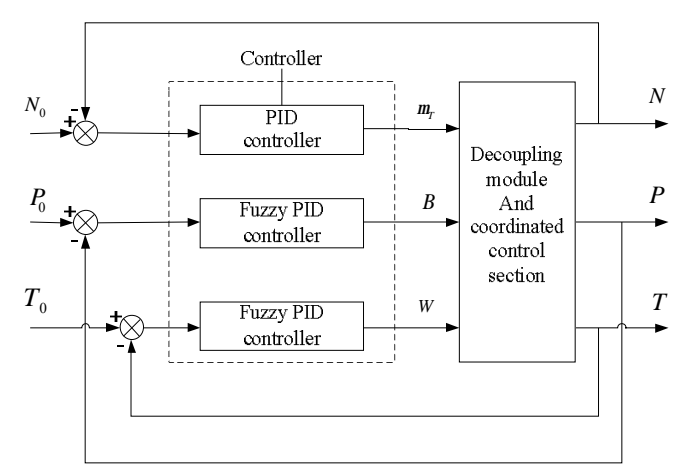

Figure 2. The structure of coordinated control system in supercritical unit

Adaptive fuzzy PID control is based on the theory of fuzzy mathematics. The conditions and the corresponding operation are described as fuzzy sets. Moreover, the empirical knowledge is saved to the knowledge base of fuzzy controller in advance. According to the output deviation of the control system and its rate of change, the PID controller parameters could be adjusted online by fuzzy inference[5]. The structure of adaptive fuzzy control system is shown in figure 3 .

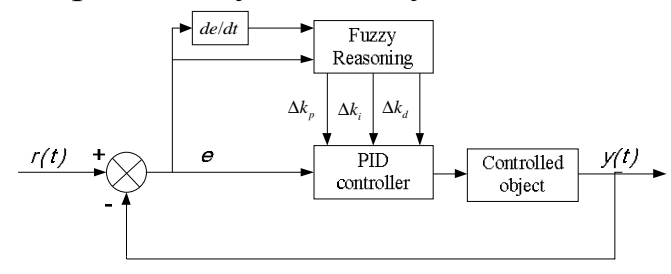

Figure 3. The structure of adaptive fuzzy control system

Based on the output deviation of the control system (compared with the given value) and its rate of change, the fuzzy inference system would export the adjusted values $\left(\Delta \mathrm{k}_{\mathrm{p}}, \Delta \mathrm{k}_{\mathrm{i}}, \Delta \mathrm{k}_{\mathrm{d}}\right)$ of the PID controller parameters $\left(k_{p}, k_{i}, k_{d}\right)$ by fuzzifying, fuzzy inference, de-fuzzifying, etc. So the PID controller could be adjusted online, and control performance could be optimized continuously. The PID controller parameters after the adjustment of the fuzzy inference system is shown as follows.

$$
\left\{\begin{array}{l}
k_{p}(k)=k_{p}(k-1)+\Delta k_{p} \\
k_{i}(k)=k_{i}(k-1)+\Delta k_{i} \\
k_{d}(k)=k_{d}(k-1)+\Delta k_{d}
\end{array}\right.
$$

\section{The analysis of control performance}

The response curve comparison of the main steam pressure and the intermediate point temperature, which is based on the conventional PID controller and the QPSO optimized fuzzy PID controller, is shown in Figure 4.
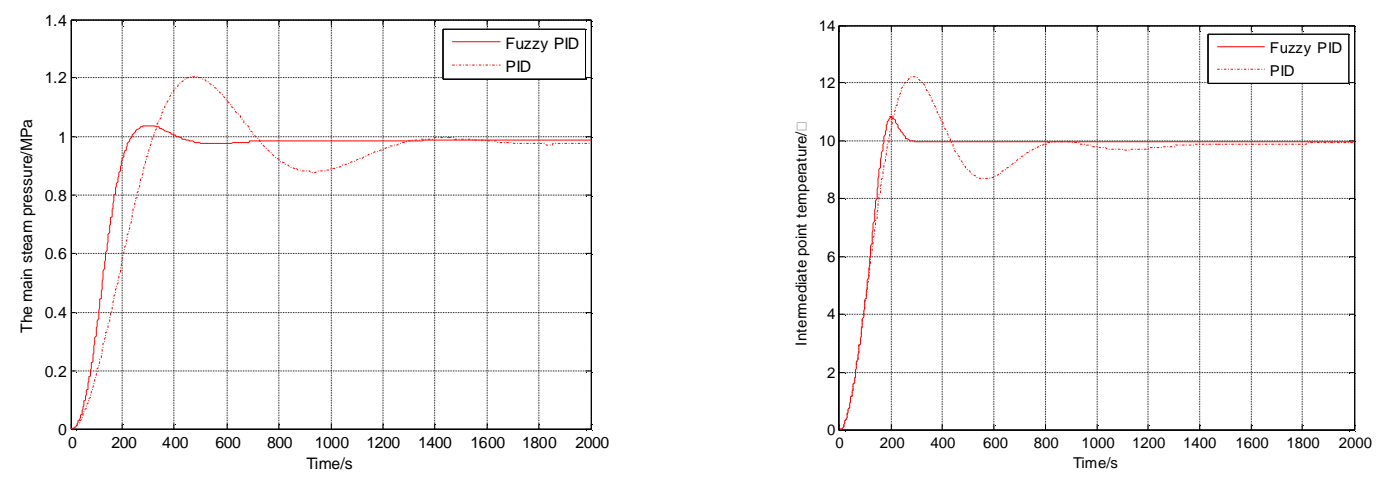

Figure 4. The response curve comparison of the main steam pressure and the intermediate point temperature 
The QPSO optimized fuzzy PID controller would adjust the proportional gain $\mathrm{k}_{\mathrm{p}}$, integral gain $\mathrm{k}_{\mathrm{i}}$, differential gain $\mathrm{k}_{\mathrm{d}}$ according to the deviation and the deviation rate of change, while the parameters of the constant PID controller are constant. From Figure 4, we could discover the difference between the QPSO optimized fuzzy PID controller and the constant PID controller. With the application of the QPSO optimized fuzzy PID controller, the overshoot of the main steam pressure response would reduce from $20 \%$ to $4 \%$, and the settling time of the main steam pressure would reduce from 1400 seconds to 600 seconds. For the intermediate point temperature response, the overshoot would reduce from $22 \%$ to $8 \%$, and the settling time would reduce from $800 \mathrm{~s}$ to $280 \mathrm{~s}$. The control performance is improved significantly.

Because the production process of a thermal power plant is complex and changeable, and there are various influence factors, the characteristics of the controlled object will change along with the change of the operation condition. When the characteristics of the controlled object change, we care about whether the performance of the QPSO optimized fuzzy PID controller is still appropriate. Thus, the simulated test, on the condition that the characteristics of the coordinated control object has changed, is conducted on Simulink platform. In this simulation process, the amplification coefficient of the transfer functions in each branch, which represents the characters of the controlled object, is increased by $50 \%$. The simulation results are shown in Figure 5.
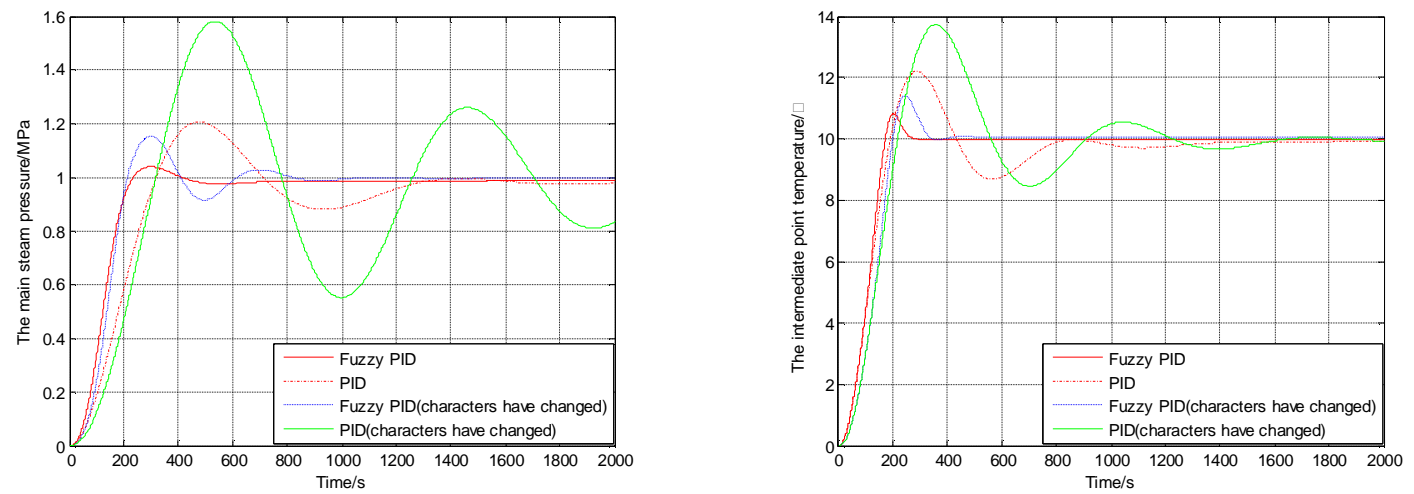

Figure 5. Response curve of main steam pressure and intermediate point temperature after change of controlled object

Based on the simulation results in Figure 5, we could notice different performance when the characters of the controlled object have changed a great deal. If the conventional PID controller is applied, the overshoot of the main steam pressure response would increase from $20 \%$ to $58 \%$, and the overshoot of the intermediate point temperature would increase from $22 \%$ to $37 \%$, and the settling time of the intermediate point temperature would increase from $800 \mathrm{~s}$ to $1300 \mathrm{~s}$. That means the conventional PID controller could not conduct timely and effective control any more. However, If the QPSO optimized fuzzy PID controller is applied, four things will occur: 1) the overshoot of the main steam pressure response would just increase from $4 \%$ to $15 \%$; 2 ) the settling time of the main steam pressure response would increase from $600 \mathrm{~s}$ to $800 \mathrm{~s} ; 3$ ) the overshoot of the intermediate point temperature response would just increase from $8 \%$ to $14 \%$; 4 ) the settling time of the intermediate point temperature response would increase from 280s to $350 \mathrm{~s}$. These will result from the QPSO optimized fuzzy PID controller adjusting its parameters, according to the deviation and the deviation rate of change when the controlled objects has changed. The data mentioned above mean the performance of the QPSO optimized fuzzy PID controller would just change slightly when the controlled object has changed greatly, and effective control is still carried out.

Moreover, there are all kinds of disturbances during the power plant operation, which would influence the control performance significantly. Therefore, whether the control system could effectively eliminate the disturbance is one of the important indexes for evaluating control system performance. When a Once-through boiler is applied in supercritical unit, and there is no drum as a 
buffer, the boiler heat storage capacity will be smaller when compared with a traditional boiler. As a result, the change of feedwater flow would directly influence the heating area proportion of heating section, evaporation section and superheat section, then the steam parameters would be led to great changes. A disturbance of feedwater flow is assumed in the follows simulation example so that we are able to analyze the capacity of eliminating disturbance in the fuzzy self-adaptation PID control system. The simulation result is shown in Figure 6.
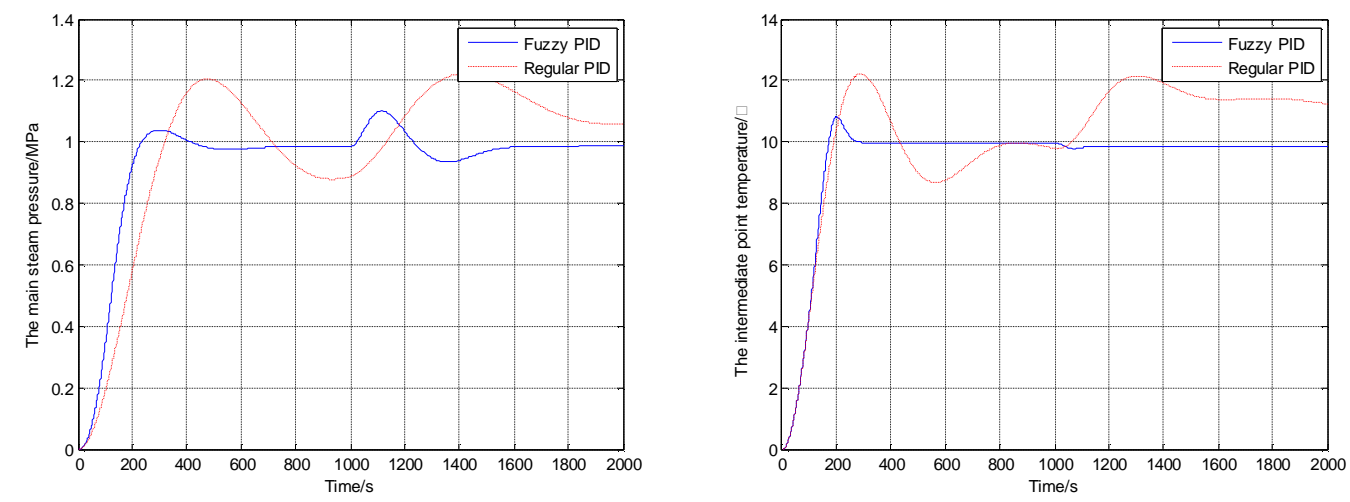

Figure 6. Response curve when the step change amplitude of feedwater flow is 80

In Figure 6, the step change amplitude of feedwater flow at 1000 second is 80 . In the fuzzy PID control system, compared with the constant PID controller, the four things will occur: 1) the overshot of main steam pressure is reduced from $22 \%$ to $9 \%$; 2) the settling time of main steam pressure is reduced to $600 \mathrm{~s} ; 3$ ) the overshot of intermediate point temperature is reduced from $20 \%$ to $3 \%$, 4) the settling time of intermediate point temperature is reduced from $1000 \mathrm{~s}$ to $150 \mathrm{~s}$. The fuzzy PID controller could greatly reduce the settling time of output response. Thus, if the fuzzy self-adaptation PID controller is applied, the influence from feedwater disturbance could be timely and effectively eliminated for main steam pressure and intermediate point temperature, and the controlled parameters would be stable around the set values.

\section{Conclusion}

According to the above simulation results, the application of the QPSO optimized fuzzy PID controller could not only improve the control performance of the PID controller but also improve the adjusting capacity for changing controlled objects. In addition, the resistance capacity to interference signal is also strengthened in the QPSO optimized fuzzy PID control system.

\section{References}

[1] Zhang YF. Thermal control technology of ultra supercritical thermal power units [M]. Beijing: China Electric Power Press, 2013.

[2] Wang J, Jiang GQ. RBF neural network control of main steam temperature based on hybrid learning algorithm [J].Thermal power generation, 2009, 38(2):28-31.

[3] Sun J, Liu X. The application prospects of intelligent PID controller in power plant process control [J]. 2012 Proceedings of International Conference on Modelling, Identification and Control, 2012: 399-403.

[4] Li GY, He XG, Yan GW, etc. Process control system [M]. Beijing: Electronic Industry Press, 2013.

[5] Shi XM, Hao ZQ. Fuzzy control and its MATLAB simulation [M]. Beijing : Tsinghua University Press, 2008. 
